\title{
CDISC SEND Pregnancy Findings Test Code Terminology
}

National Cancer Institute

\section{Source}

National Cancer Institute. CDISC SEND Pregnancy Findings Test Code Terminology. NCI

Thesaurus. Code C124324.

Terminology associated with the pregnancy findings test code codelist of the Clinical Data Interchange Standards Consortium (CDISC) Standard for the Exchange of Nonclinical data (SEND). 